\title{
ULTIMATE PERFORMANCE OF THE LEP RF SYSTEM
}

\author{
P.Brown, O.Brunner, A.Butterworth, E.Ciapala, H.Frischholz, G.Geschonke, E.Peschardt, J.Sladen, \\ CERN, Geneva, Switzerland
}

\begin{abstract}
:
The LEP Superconducting RF system reached its maximum configuration of 288 four-cell cavities powered by 36 klystrons in 1999 . In 2000 , this system, together with 56 cavities of the original copper RF system, routinely provided more than $3630 \mathrm{MV}$, allowing the beam energy to be raised up to $104.5 \mathrm{GeV}$. This not only required operating the cavities more than $15 \%$ above their design gradient, but has also demanded a very high operational reliability from the entire system. This paper will describe the operation of the LEP RF system during 2000 , including new features, operational procedures and limitations.
\end{abstract}

\section{INTRODUCTION}

In preparation for the LEP energy upgrade, the first prototype superconducting (SC) RF module with four cavities was tested in LEP in September 1992. Following further development of this technology, installation of the series production cavities started in 1995 . By October 1995 in addition to the existing 128 copper cavities a total of 64 SC cavities were operational, allowing a gradual increase in beam energy from $45 \mathrm{GeV}$ to $70 \mathrm{GeV}$. In 1996 with 144 SC cavities, $\mathrm{W}^{ \pm}$physics started at $80.5 \mathrm{GeV}$ per beam. Subsequently more SC cavities were added, initially complementing and then, when all available space was filled, partially replacing, the original copper system. The final configuration is given in table 1. The last two years of LEP physics centred on the search for the Higgs boson, consequently the highest possible beam energy at good luminosity became the main priority. The performance of the RF system was pushed far beyond its original specifications. The evolution of the nominal and the operationally available RF voltage since 1995 as well as the beam energy is shown in figure 1 . Figure 3 shows the achieved accelerating gradients with increasing beam energy.

The circumferential voltage $U_{0}$ required to replace the synchrotron radiation losses per turn at a beam energy $E$ is given approximately by:

$$
U_{0}[\mathrm{eV}]=28.6 * E^{4}[\mathrm{GeV}]
$$

At $104.5 \mathrm{GeV}$ the two counter rotating beams lose $3.4 \mathrm{GeV}$ per turn, corresponding to $3.2 \%$ of their energy. In order to keep the beams stable, an over voltage of about $10-15 \%$, depending on the quantum lifetime, is required. With a total accelerating voltage of about 3.6 GV the LEP RF system was the biggest acceleration system built so far in a storage ring.

The nominal circumferential voltage given in figure 1 is calculated from the gradients specified at production of the cavities. Throughout the upgrading programme there was continuous pressure for the highest possible beam energy.

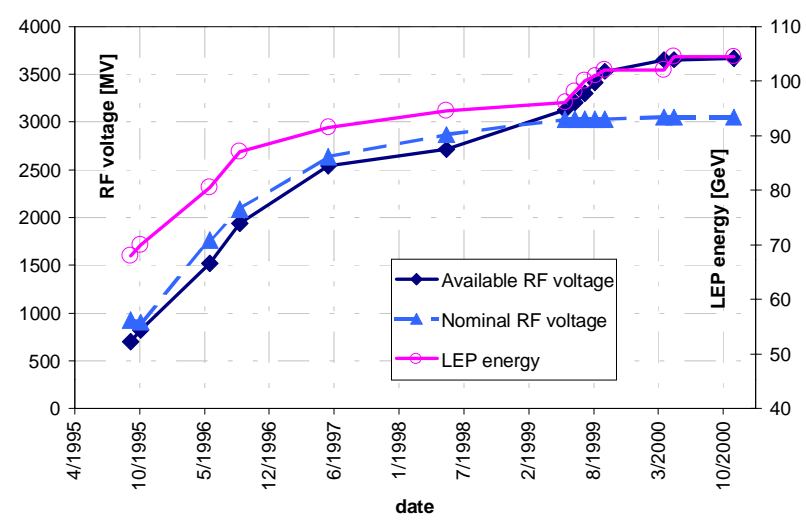

Figure 1.: Beam energy, available and nominal RF voltage from 1995 until 2000.

During the last two years the beam energy could be significantly increased by several measures [1]: Increasing the bending radius by using the corrector magnets, changing the horizontal damping partition number, implementing special operational procedures and, principally by increasing the accelerating gradient of the SC cavities.

This report describes the final performance of this system and the measures taken to achieve it.

\section{SYSTEM DESCRIPTION}

The $\mathrm{Cu}$ system is described in [2]. Details of the SC system can be found in [3] and [4] and consequently only the main features are given below. The $352 \mathrm{MHz}$, standing wave, four-cell, SC cavities, were mounted in groups of four in one common cryostat, called a "module". They were cooled in a bath of liquid He at $4.5 \mathrm{~K}$, a total cooling capacity of $53 \mathrm{~kW}$ at $4.5 \mathrm{~K}$ was available [4]. The first 16 superconducting cavities were constructed of solid $\mathrm{Nb}$, all later ones were made by sputter-coating a $\mathrm{Cu}$ substrate with a thin layer of $\mathrm{Nb}$.

Each 1.3 MW klystron fed eight cavities in parallel, two klystrons shared one common high voltage DC power supply in one "RF unit". For reasons of compatibility the layout of the RF plant for the SC system was essentially the same as for the $\mathrm{Cu}$ system. The RF power was controlled by a "Voltage Loop", which maintained the sum of the eight individual cavity voltages at the requested level independent of beam loading. For most RF units scalar sum detection was used, however in some units a vector sum was implemented. Table 1 gives the main parameters of the final configuration. 
The cavities were installed in the straight sections on either side of the four even numbered interaction points of LEP.

Table 1 The LEP RF system in 2000

\begin{tabular}{|c|c|}
\hline number of $\mathrm{Cu}$ cavities & 56 \\
\hline accelerating voltage per cavity & $2.5 \mathrm{MV}$ \\
\hline active length of 4-cell cavity & $1.7 \mathrm{~m}$ \\
\hline number of solid $\mathrm{Nb} \mathrm{SC}$ cavities & 16 \\
\hline design gradient & $5 \mathrm{MV} / \mathrm{m}$ \\
\hline number of sputtered Nb SC cavities & 272 \\
\hline design gradient & $6 \mathrm{MV} / \mathrm{m}$ \\
\hline number of klystrons (1/1.3 MW each) & 44 \\
\hline
\end{tabular}

\section{SC CAVITY PERFORMANCE}

The solid $\mathrm{Nb}$ cavities were conditioned up to their nominal design gradient of $5 \mathrm{MV} / \mathrm{m}$ and operated up to 4.5 MV/m with beam. The average gradient of the sputtered SC cavities was significantly increased above the design gradient of $6 \mathrm{MV} / \mathrm{m}$, after a number of reliability issues of ancillary equipment had been addressed and limitations had been removed [4].

A few cavities degraded in performance during operation. Since 1998 two modules were temporarily removed from the tunnel for repair of individual cavities during the winter shut-downs. Six cavities could be recovered by rinsing with ultra-pure water, in one case the $\mathrm{Nb}$ film had to be re-sputtered.

A hard limitation of cavity gradient was field emission, leading to local heating of the cavity walls and a sharp rise in the pressure of the $\mathrm{He}$ bath, which triggered the safety interlock. In some cavities field emission lowered the $\mathrm{Q}_{0}$-value and cavity dissipation exceeded the available cryogenic cooling power, leading to a limitation of the accelerating field. The maximum cooling capacity per cavity of approximately $200 \mathrm{~W}$ is given by the cryostat design.

Apart from aggressive cavity conditioning, the increase in gradient was achieved by removing a number of limitations, described below.

\subsection{Field Equalisation}

Eight cavities were connected to a common klystron, with no control of RF power to individual cavities.

The cavities were equipped with fixed power couplers. Due to mechanical tolerances and variations of field flatness of the four cells, the external Q-factor $\mathrm{Q}_{\mathrm{ext}}$ of the power couplers varied around the nominal value of $2 * 10^{6}$ by $\pm 20 \%$. The effect of extreme $\mathrm{Q}_{\mathrm{ext}}$ of certain cavities, leading to particularly high fields, and therefore limiting the whole group, was corrected by inserting $\lambda / 4$ plates of appropriate thickness and distance from the power couplers, into the waveguide. This technique was also applied to increase the field of some low-field cavities.

Each cavity had its own frequency tuning circuit using as reference signal the forward signal of a directional coupler upstream of the power couplers. The reflection from the cavities in the strongly beam loaded regime of the SC cavities varies between almost $100 \%$ without beam and $0 \%$ at nominal beam current. Insufficient directivity of the directional couplers can lead to beam current dependent changes of cavity tuning. Therefore, the directivity of all these couplers was adjusted to better than $30 \mathrm{~dB}$.

In order to achieve equal fields with maximum beam current of about $6 \mathrm{~mA}$, the electrical length of the waveguides feeding a group of eight cavities had to be adjusted to a precision of about 3 degrees on all cavities.

\subsection{Cavity Oscillations}

Ponderomotive oscillations had been a major limitation of cavity performance [6]. In the last year of operation this problem was removed by implementing an active damping system on all cavities, which made use of the existing tuning system [4].

\subsection{Cavity Conditioning}

During the winter shut-downs the whole system was brought to room temperature, requiring reconditioning each year. The criteria for conditioning were local vacuum in the power couplers and $\mathrm{x}$ - and $\gamma$ - radiation from the cavities.

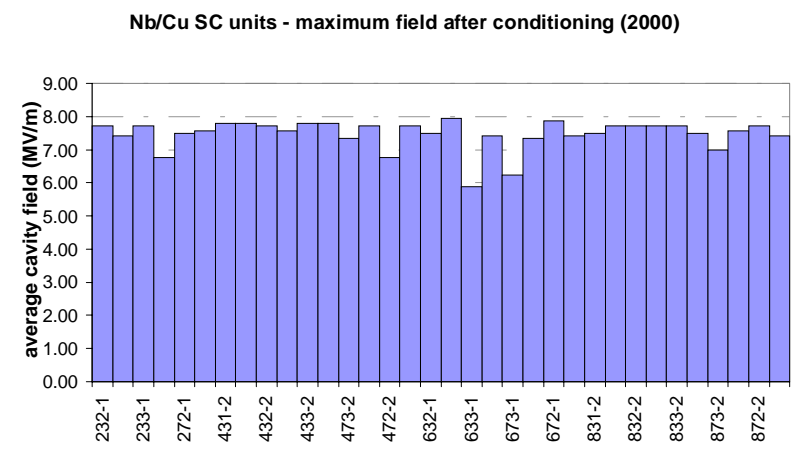

Figure 2: Average accelerating gradient of RF units with sputtered SC cavities after conditioning in 2000.

Both continuous (cw) and pulse conditioning with pulse length varying between $10 \mathrm{~ms}$ and $100 \mathrm{~ms}$ and duty cycle between 1 and $10 \%$ were used. A smooth switchoff of the RF pulse was implemented to avoid excessive peak power reflections from the cavities causing arcs in the waveguides. The average gradients of all groups of eight cavities after conditioning are shown in figure 2. The goal for conditioning in 2000 was $7.5 \mathrm{MV} / \mathrm{m}$ average. For stable operation with beam the total gradient was about $5 \%$ lower than the maximum achieved during conditioning. He processing had been applied in isolated cases in the past with mixed results. The inherent risks of processing in situ using klystrons as RF power sources with strongly overcoupled power couplers were considered too high for systematic application of this technique and it was no longer applied in the LEP tunnel. 


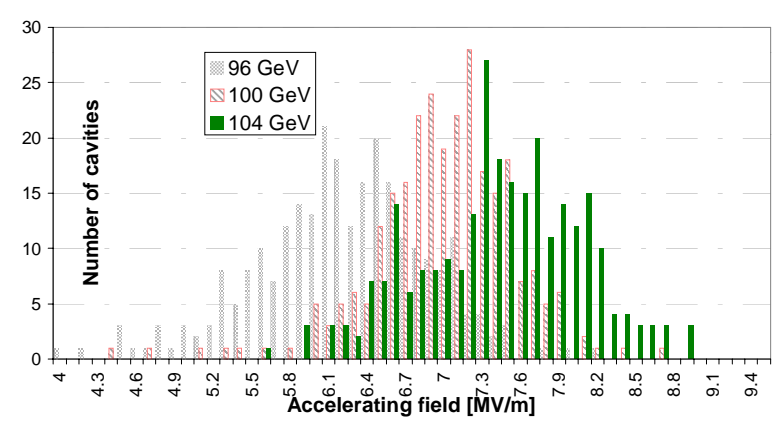

Figure 3.: Histogram of cavity gradient distribution for three different maximum beam energies in 1999 and 2000.

During the year the maximum accelerating field for each RF unit was set individually, such that stable maximum voltage operation with beam was possible. This level was regularly adjusted. Some units had to be temporarily reduced, but overall most were slowly increased. By permanently running at or near the limit, the maximum total accelerating voltage was increased to a record of $3666 \mathrm{MV}$. This in spite of the loss of four cavities permanently detuned because of damage and another three cavities which had to be partly detuned to achieve stable operation.

\section{OPERATIONAL PROCEDURES AND SYSTEM PERFORMANCE}

The exploitation of the highest cavity gradients was only possible by a new LEP running scenario and modified RF operational procedures.

In previous years, the number of different beam energies was limited by the LEP physics experiments to three. However, during 2000, the accumulation of luminosity at the highest possible energy resulted in an almost continuous distribution of energies during physics coasts.

Trips of the RF interlock system occurred mainly due to field emission, on a statistical basis. An RF trip lost the voltage from eight cavities, about $100 \mathrm{MV}$, corresponding to $0.8 \mathrm{GeV}$ beam energy. A trip of a HV supply (two klystrons) lost $200 \mathrm{MV}$ and $1.6 \mathrm{GeV}$.

The beam energy was determined by the available RF voltage on a fill by fill basis. At the beginning of a physics coast the beam energy was chosen such that the beam survived the loss of 16 cavities, i.e. between 102.0 and $102.8 \mathrm{GeV}$. During the coast, the beam energy was "mini-ramped" by $0.8 \mathrm{GeV}$, reducing the margin to a trip of one klystron. Another miniramp of $0.8 \mathrm{GeV}$ at the end of the coast reduced the quantum lifetime such, that a klystron trip would cause the loss of the beam.

The average time between RF trips was 14 min and recovery normally took about 2 min, except in the case of equipment failure. The average length of a physics coast was 1.5 hours.

A GPS timing based diagnostics system was introduced, which allowed precise determination of the sequence of all unit trips, distributed over $26.7 \mathrm{~km}$. The system could distinguish losses of the circulating beams due to RF trips or due to other reasons. This system proved very important for efficient fault finding.

At injection energy the beam induced electric field in the cavities was much higher than the required cavity field, therefore particular care was taken to correct tuning of the cavities, since small errors could lead to a run-away of the cavity tuning. In particular, during the power ramp the tuning state of cavities was checked and the maximum field of RF units was adjusted dynamically.

Permanent operation of the system at - and sometimes beyond - safe limits caused damage to components of the RF plant. In particular arcing in waveguides led to destruction of several components and to damage of three circulators.

\section{CONCLUSION}

The performance of the RF system contributed principally to the increase in LEP centre of mass energy of $7 \mathrm{GeV}$ since 1999, and allowed the Higgs boson discovery limit $(5 \sigma)$ to be pushed to $113 \mathrm{GeV} / \mathrm{c}^{2}$ [7]. With the shut-down at the end of 2000 LEP operation came to an end. The RF system is now dismantled.

\section{ACKNOWLEDGEMENTS}

The successful operation of the LEP RF system and its ultimate performance was only possible due to the dedication of all members of the SL LRF group who participated in the daily performance meetings and in the very demanding stand-by service. We are pleased to also acknowledge the important contributions from all other groups involved in RF system operation, in particular the cryogenics and the operations teams.

\section{REFERENCES}

[1] R.Assmann, "LEP Operation and Performance with Electron - Positron collisions at $209 \mathrm{GeV}$ ", Proc. XI Chamonix workshop 2001, CERN-SL-2001-003 DI

[2] G.Geschonke, S.Hansen, "Status and Performance of the LEP RF System", Conf. on High Energy Accelerators, Tsukuba 1989, Part Acc. 28(1990), p. 779

[3] D.Boussard, "Performance of the LEP2 SRF System", PAC, Vancouver 1997, p. 2879

[4] P.Brown, O.Brunner, A.Butterworth, E.Ciapala, H.Frischholz, G.Geschonke, E.Peschardt, J.Sladen, "Performance of the LEP200 Superconducting RF System", 9. Workshop on RF Superconductivity, 1999 Santa Fe, LA-13782-C, p. 1

[5] M.Sanmarti, Ph.Gayet, S.Claudet, "Cryogenic Issues for LEP in 2000", LEP -SPS Performance ws. Chamonix X, CERN-SL-2000-007 DI, p.226

[6] D.Boussard, P.Brown, J.Tückmantel, "Electroacoustic Oscillations in the LEP SC Cavities", EPAC Sitges 1996, p. 187

[7] P.Janot,CERN/EP, private communication 